\title{
WATERLOO BRIDGE MONITORING: COMPARING MEASUREMENTS FROM EARTH AND SPACE
}

\author{
S. Selvakumaran ${ }^{1 *}$, G.T. Webb ${ }^{2}$, J. Bennetts ${ }^{2}$, C.R. Middleton ${ }^{1}$ and C. Rossi ${ }^{3}$ \\ ${ }^{1}$ Department of Engineering, University of Cambridge, Trumpington Street, Cambridge, United Kingdom \\ ${ }^{2}$ WSP, Devonshire Square, London, United Kingdom \\ ${ }^{3}$ Satellite Applications Catapult, Electron Building, Fermi Avenue, Harwell, United Kingdom \\ * Corresponding author
}

\begin{abstract}
Satellite radar imagery is becoming more accessible, and at improving spatial and time resolutions. Interferometric Synthetic Aperture Radar (InSAR) has the capability to provide wide-area, high density, millimetre-level displacement measurements (obtained even in darkness and adverse weather conditions). This paper examines advances in InSAR satellite measurement technologies to understand their relevance, utilisation, and limitations for bridge monitoring. Waterloo Bridge is presented as a case study to explore how InSAR data sets can be combined with traditional measurement techniques including sensors installed on the bridge and automated total stations. A novel approach to InSAR bridge monitoring was adopted by the installation of physical reflectors at key points of interest on the structure, in order to supplement the bridge's own reflection characteristics and ensure that the InSAR measurements could be directly compared and combined with in-situ measurements. The interpretation and integration of InSAR data sets with civil infrastructure data is more than a trivial task. Key assumptions and their implications for data interpretation are discussed, with a view to how this technology could be employed in the future.
\end{abstract}

\section{Introduction}

Asset maintenance for bridges in most countries comprises a monitoring scheme centred on periodically scheduled visual inspections to assess asset condition, performance and levels of deterioration. Once a problem has been identified, there may be further inspections or the installation of a structural health monitoring scheme, involving sensors, surveying and other quantifiable measurements. Installing structural monitoring systems does not always yield value and is not a financially viable or practical option for all assets (Webb et al., 2014). Furthermore, the installation of measurement devices and collection of data is not always straightforward.

Interferometric synthetic aperture radar (InSAR) techniques are well established within the earth observation sector, with radar satellites being regularly launched from the 1990s onwards. Satellites passing over the earth take synthetic aperture radar (SAR) images. A stack of these images acquired at different time instances can be processed using differential SAR interferometric techniques where subtle changes of the range distance between the acquisitions can be detected. These techniques provide a remote means of monitoring millimetrescale changes over time of multiple discrete points over large spatial areas (Bamler and Hartl, 1998; Ferretti et al., 2001).

These stacking techniques were originally used for Earth science applications such as the monitoring of earthquakes, volcanoes and other large objects. SAR satellites cover the entire Earth (polar orbit) and are active imaging systems (they emit and collect radar signals). They are therefore able to operate irrespective of day or night, and even when there is cloud cover. In more recent years, the frequency of acquisitions has increased for a given satellite system (by putting multiple satellites within the same orbit) and the spatial resolution of the imagery collected has become much finer, opening this technique for use in infrastructure monitoring.

On the topic of bridge monitoring, the literature provides evidence that InSAR can be used to monitor different features of bridge behaviour, such as ground settlement at pier locations or seasonal thermal expansion (Fornaro et al., 2012; Lazecky et al., 2017; Selvakumaran et al., 2018; Sousa and Bastos, 2013). Many of the bridge structures studied in the literature are large scale metal bridges which make use of natural reflectors created by the structure's form and geometry. The studies focus on the interpretation of SAR measurements, often without in-situ measurement data.

The use of InSAR for bridge monitoring has the potential to supplement existing visual inspection schemes with more frequent data collection readings, with remote access. In addition, traditional surveying and monitoring techniques could be supplemented with additional insights into the behaviour of the bridge, as well as the wider surrounding region.

In this paper Waterloo Bridge is presented as a case study in investigating the potential for satellite monitoring data to be used to support bridge management activities. Corner reflectors and target prisms for automated total station measurements have been installed at key points of interest as part of a wider structural monitoring system, and displacement data from the systems are compared and studied. 


\section{Principle of InSAR techniques}

The imaging geometry of a radar system (with key parameters illustrated below in Figure 1) is different from the framing and scanning systems commonly employed for optical remote sensing (such as those employed for Google Earth imagery). The satellite travels in the flight direction. The nadir is the point on the Earth's surface directly below the satellite sensor as it progresses along its line of flight (this progression is marked below as the nadir track). The radar signal is emitted obliquely to the flight direction, and this illuminates a section of the Earth's surface with swath width, as marked. This form of viewing geometry is known as 'side-looking' because the satellite looks sideways at an oblique angle from its flight path.

Figure 1 Graphic to illustrate key parameters in SAR imaging geometry.

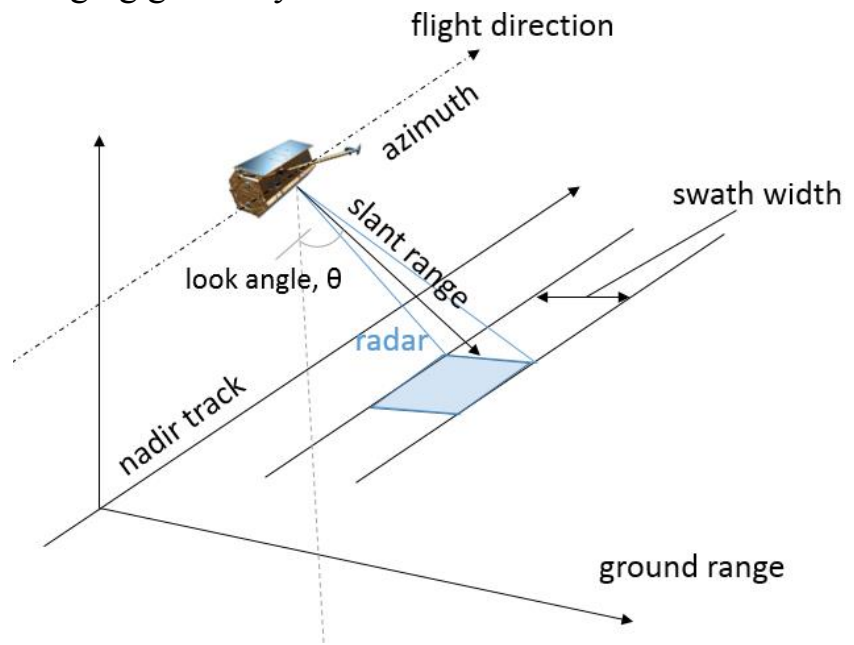

There are several current and historical satellites that can provide SAR data (collected at different wavelengths depending on the satellite). These satellites differ not only in radar frequency and wavelength, but also in polarisation, temporal resolution, spatial resolution, swath, availability of data and cost (Ferretti et al., 2007a). Satellites with the objective of SAR data collection are designed to follow a nearpolar orbit around the earth. The satellite travels northwards on one side of the Earth (ascending pass) and then toward the southern pole on the second half of its orbit (descending pass). In conjunction with the earth's own orbit (east-west) this orbit allows the satellite to cover most of the earth's surface over a certain time period. Shorter wave X-band satellites (rather than C- or L-band) provide more accurate displacement results, and data from such satellites is currently accessible through commercial satellites.

SAR imaging is the production of a digital SAR image that is made up of a two-dimensional array formed by columns and rows of 'pixels'. These SAR pixels are associated with an area on the earth's surface (the size of the resolution cell varies with each satellite). Each pixel gives a complex number that carries amplitude and phase information about the microwave field backscattered by all the 'scatterers' within the corresponding resolution cell projected on the ground. Image resolution in different directions is achieved by transmitting various types of coded waveforms and applying various forms of filtering techniques (Simons and Rosen, 2007). The radar backscatter value depends on the local topography, including rocks, vegetation, buildings etc., which are the scatterers of the signal. The SAR image contains a measurement of the amplitude of the radiation backscattered toward the radar by scatterers contained in the SAR resolution cell. It should be noted when interpreting SAR imagery that it is affected by surface characteristics such as surface roughness, vegetation density and soil moisture, rather than the 'colour' used to interpret the equivalent optical image.

Exposed rocks and urban areas generally show strong amplitudes, whereas smooth flat surfaces (like quiet water basins) show low amplitudes due to the radiation being mirrored away from the radar collector. SAR amplitude images are generally visible as a grey scale image (as shown later in Figure 4), and these amplitudes are visible through brightness; bright pixel areas indicate strong backscattered radiation (e.g. urban areas with hard, sharp surfaces), whereas darker pixels correspond to low backscattered radiation (e.g. standing bodies of water). For this reason, water bodies are often quite easy to identify in SAR imagery.

The radar waves transmitted from the satellite travel to the scatterers on the earth's surface and then return to the satellite to form the SAR images. As the scatterers are at different distances from the emitter on the satellite (they have different slant ranges - illustrated in Figure 1), different delays are introduced into the reflected return signal. Interferometry relies on "the constructive and destructive interference of electromagnetic waves from sources at two or more vantage points to infer something about the sources or the relative path length of the interferometer" (Simons \& Rosen, 2007). The satellite generates the outgoing SAR signal (with known phase) which can then be compared to the phase of the return signal which is dependent on the distance to the ground (see Figure 2 below). The complex phase vector information of one image is multiplied by the complex conjugate phase vector information of another image to form an "interferogram". This results in the common backscatter phase in each resolution element being cancelled whilst leaving a phase term proportional to the differential path delay. Thus, an "interferogram" is an image with each pixel comprising a phase difference (from 0 to $2 \pi$ radians) between two distinct SAR snapshots of a given resolution cell. 
Figure 2 Simplified depiction of InSAR principle.

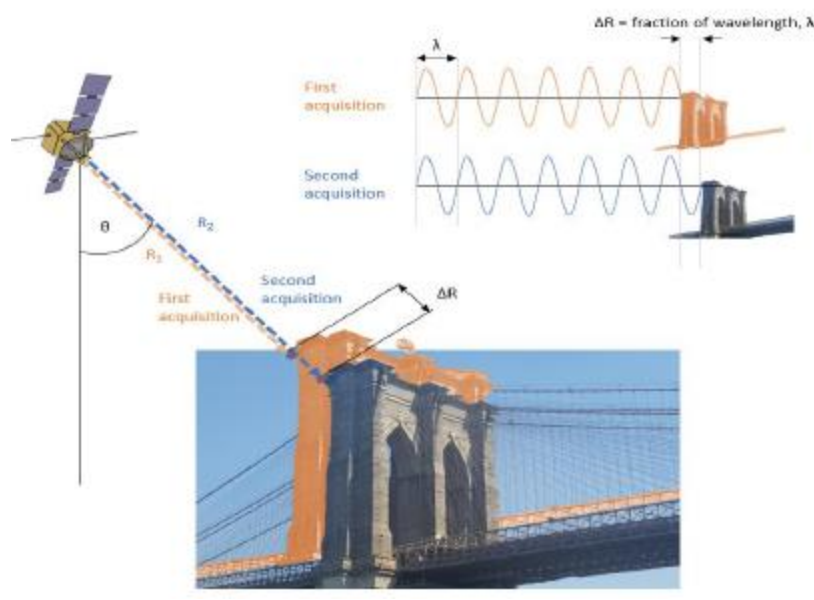

\section{Monitoring of Waterloo Bridge}

\subsection{Waterloo bridge structure}

Waterloo Bridge is a $434 \mathrm{~m}$ concrete bridge carrying the A301 across the River Thames in London. The bridge comprises two reinforced concrete, multi-cell box girders and a concrete deck. It has five main spans and is nominally symmetric about its centreline. In each half the box girders and deck are continuous over two spans, with cantilever projections beyond the abutment piers and into the central span, where they support a central drop-in section. In 1981 the bridge was designated as a Grade II* Listed structure by Historic England.

\subsection{Background to monitoring work}

The bridge responds to changes in temperature by expanding or contracting over its $434 \mathrm{~m}$ length. This daily and seasonal change in length of the structure is accommodated through four expansion joints, one at each abutment and one at each end of the central drop-in span. Recent inspections have revealed a number of concerns relating to the performance of the bridge's articulation, prompting further work to investigate the bridge's current condition and develop potential remedial solutions if necessary.

The roller bearings supporting the bridge's central drop-in span are of an unusual design. Recent inspections have highlighted significant deterioration, with parts of these bearings becoming detached, prompting safety concerns for river traffic passing under the bridge. There are also concerns that the bearings and lateral restraint members across the joints may have locked up due to corrosion, silt build-up, and dislodged components. If true, this would have the consequential effect of changing the bridge's articulation, introducing additional stresses into the structure's deck and piers when the bridge expands and contracts due to temperature variations. The approach slabs at both ends of the bridge are supported by bearings on the ends of the approach viaducts. The original segmental roller bearings at the north and south abutments were replaced with elastomeric bearing pads in 2006 and 2010 respectively. However, some of the replacement bearings have failed and displaced from their supports, suggesting that they are experiencing movement ranges greater than expected during their design. It is likely that the bridge's approach viaducts will also respond to temperature changes and may therefore be contributing to the failure of the elastomeric bearings.

\section{Deployed monitoring systems}

\subsection{Deployed traditional monitoring systems}

An automated remote monitoring system comprising 48 temperature sensors and 20 displacement transducers was installed within the bridge deck. Additionally, two Automated Total Stations (ATS) were installed looking at either side of the bridge to monitor 12 reflective prisms (six prisms have been attached to each side of the structure, one at each of the bridge's piers, and one at each end of the central drop-in span). Additional prisms were installed on sites outside of the bridge as reference points. Measurements from the temperature and displacement gauges were taken simultaneously every 1 second, and from the ATS every 5 minutes.

Figure 3 ATS target prism locations on the bridge (base optical image provided by Google Earth).

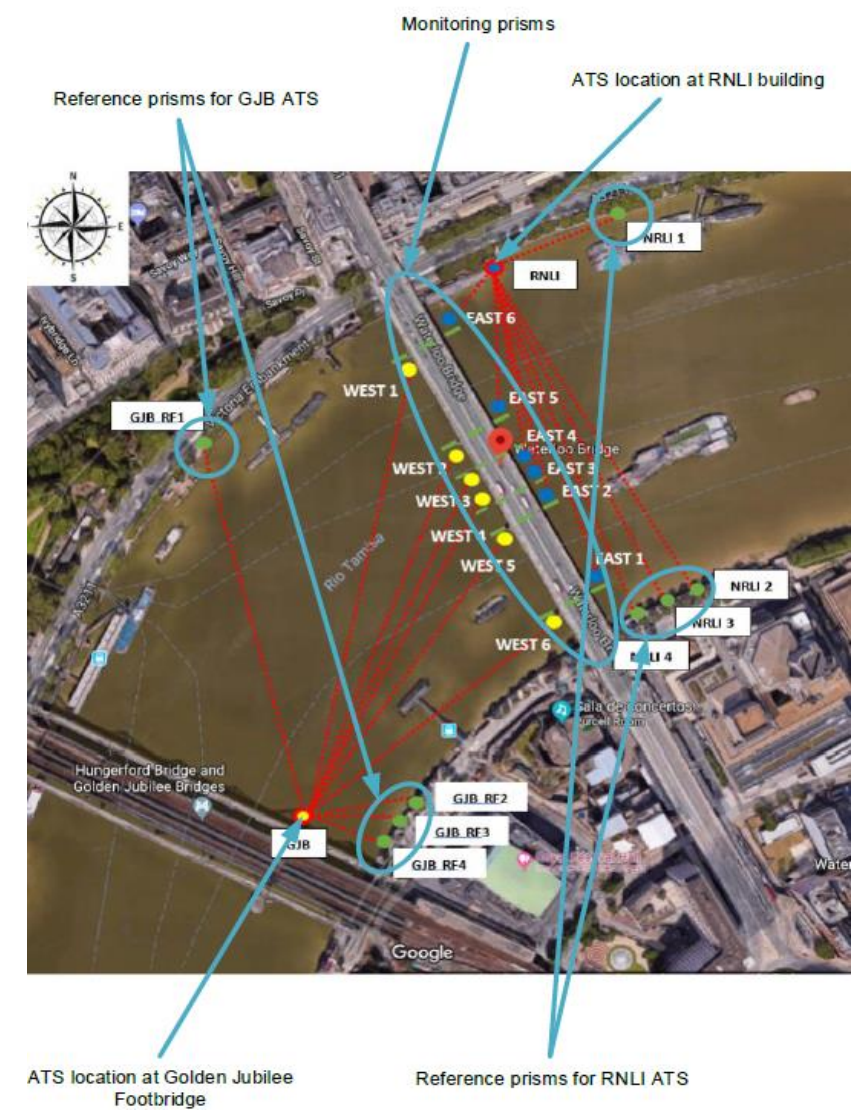

\subsection{InSAR monitoring installation}

\subsubsection{Data sets}

Satellite images were collected by the TerraSAR-X satellite. Each time the satellite passes over London either from North 
to South (denoted 'descending') or from South to North (denoted 'ascending') radar imagery is captured. TerraSAR-X Stripmap mode images $(3 \mathrm{~m} \times 3 \mathrm{~m}$ ground resolution $)$ were programmed for readings every 11 days in either direction from December 2017 until October 2018. An 11-day interval is the shortest repeat cycle available for this satellite. SAR satellite constellations all currently have revisit periods in the order of days, rather than hours.

For differential SAR interferometry, a Digital Elevation Model (DEM) is required to remove topographic information common to all images, which then leads to differential measurements. Shuttle Radar Topography Mission (SRTM) data with resolution of 3 arc-seconds $(90 \mathrm{~m})$ was used. The data from this satellite is provided by NASA free of charge. This data is much coarser in resolution than the SAR data (the bridge is not represented within this image) but this is not a fundamental input in this processing of differential movement at discrete corner reflector locations (an ellipsoid representing Earth curvature could also have been used).

\subsubsection{Methodology}

Interferometric techniques for deformation monitoring exploit the information contained in the phase of at least two complex SAR images acquired at different times over the same area, by forming an interferogram (Crosetto et al., 2016).

Persistent or Permanent Scatterer Interferometry (PSI) uses reflectors whose response to the radar is dominated by a strong reflecting object and is constant over time and makes use of classical Differential Interferometry Synthetic Aperture Radar (DInSAR). A procedure for the identification and exploitation of stable permanent scatterers was developed by Ferretti et al. and results in displacement accuracy in the line of sight of the satellite of the order of millimetres (Ferretti et al., 2007b, 2000). The technique relies on analysing pixels which remain fixed, or coherent over a sequence of interferograms.

For many structures, no installation is required, as the radar satellite makes use of natural reflectors (persistent scatterers, or PS points) but in this case, the bridge was augmented with a physical installation for two reasons:

a) the presence of very few natural PS points

b) for scientific research in order to create PS points at known locations

One limitation of the InSAR technique is that some interpretation is required in understanding the output. Points that naturally reflect are selected, and very often, it is not clear which object within the pixel is causing the reflection. Filtering algorithms can deal with the speckle which occurs in SAR images due to the coherent sum of many elemental scatterers within a resolution cell, but for the final PSI points selected it is not always clear what the corresponding real-life object acting as a reflector is. Additionally, some structures do not reflect radar well at all.

\subsubsection{Corner reflectors}

Corner reflectors can be used to artificially create a reflective point, but the reflector must be suitably designed, and its effectiveness is dependent on the radar wavelength being used and the background clutter from other reflectors in the vicinity. There are different types of reflectors (such as a flat plate, a dihedral made of two plates forming a corner, or corners in various shapes). A trihedral radar reflector, commonly known as a "corner reflector", facilitates a triple-bounce of the incident radar energy from three mutually orthogonal plates such that the incoming radar wave emitted from the satellite is reflected back to the satellite receiver. Corner reflectors are the most commonly used type of radar target deployed for persistent scatterer InSAR (Doerry, 2008). The corner reflector becomes the dominant scatterer in a resolution pixel and is much stronger than echoes from dimmer distributed scatterers within the pixel, often referred to as "clutter". The literature identifies several theoretical and experimental studies on corner reflectors (Crosetto et al., 2013; Garthwaite et al., 2013; Sarabandi and Tsen-Chieh Chiu, 1996). There have also been studies on ground based interferometric radar measurements of bridges where corner reflectors are installed on the bridge and a ground sensor emits radar and collects radar reflections (Gentile and Bernardini, 2008).

Figure 4 Annotated SAR amplitude image with bridge marked and corner reflector installation appearing as bright points.

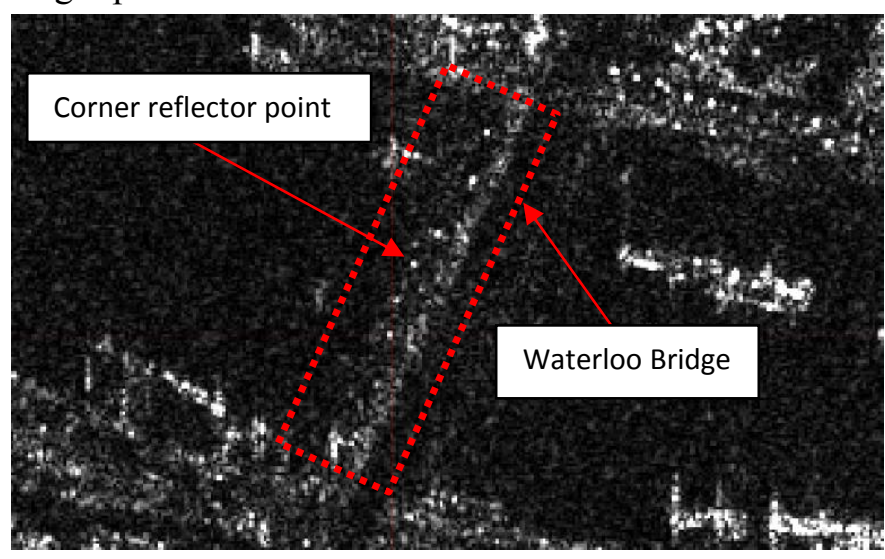

The SAR image of the bridge was assessed before planning for the study, and it was noted that the concrete structure of the bridge provided few natural strong reflections, and the noise of the signal from other points on the bridge was relatively low. The corner reflector was sized in terms of its radar cross section (RCS, a measure of the reflectivity) to be significantly higher than the background clutter (Figure 4), but small enough to be mounted on the bridge. In Figure 4 the bridge is the object crossing the dark area (the River Thames, water bodies correspond to low backscattered radiation). The corner reflector material was chosen to be aluminium, given that is light weight and does not suffer from corrosion as badly when compared to steel. Pre-fabricated perforated sheeting also reduces RCS but facilitates drainage (flooding of the reflector would have a greater reduction in RCS than the introduction of 
holes), reduces wind load, reduces overall weight and promotes self-cleaning of dirt and other deposits.

When deployed, the corner reflector must be orientated such that the azimuth and elevation of the boresight vector (originating from the intersection of the three triangular plates) is directed in the SAR satellite's line of sight (LOS). This orientation was estimated using the Systems Tool Kit (STK) modelling environment by input of the corner locations and modelling of the TerraSAR-X positioning over the site for the acquisition period, using the method described by Garthwaite et al. (2015) to account for the orbit and look angle of the SAR sensor to position and orientate the corner reflectors.

The reflectors installed in this study (an example of which is seen in Figure 5) are much smaller than those used in calibration studies or in field deployments in the literature. Smaller scale reflectors were required to gain permission for installation. As the theoretical calculation suggested that these would be seen within the imagery, they were trialled for the first time in such an application for this study, to assess suitability.

Figure 5 Aluminium corner reflector (circled) installed on bridge pier alongside ATS prism targets.

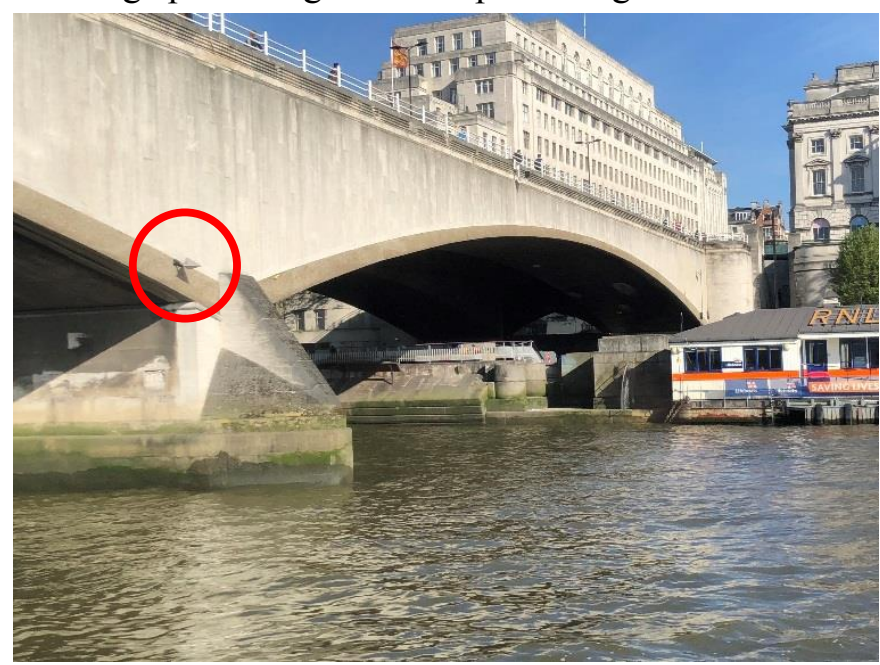

\section{Data interpretation of traditional and satellite monitoring systems}

\subsection{Limitations of the traditional ATS monitoring}

The ATS monitoring was set up to take line of sight readings to all bridge prisms, relative to stable reference points. This was to allow movements of the bridge to be distinguished from any movements of the abutments or approach viaducts (which it was not possible to instrument with displacement gauges). Raw data from ATS monitoring system comprises angle and distance measurements from the ATS location to each of the prisms. Every 2 hours the ATS observes each of its four reference prisms. Assuming that the coordinates of the reference prisms are fixed the ATS calculates its current position. Due to measurement errors the angle and distance measurements from the four reference prisms will not all result in the same answer, so a Least Squares adjustment is carried out to select the most likely position. Every 5 minutes the ATS observes each of the monitoring prisms and calculates their coordinates relative to its current location.

The reference prisms were installed on the river walls near to the bridge. The river walls elsewhere in central London are known to be affected by the height of the tide, and this is shown within the ATS data (for which the reference prisms' movements can be shown to move in strong correlation with tide). Since movements of the river walls appear to be similar in magnitude to movements of some parts of the bridge it is not possible to remove the effects of this error from the data, hence the absolute coordinates of all ATS monitoring prisms cannot be relied upon. ATS measurement readings for the bridge have been calculated as being relative to the southernmost prisms either side of the bridge, and longitudinal movement of the bridge has been measured relative to this point.

\subsection{Initial results of InSAR processing}

InSAR processing of images of the bridge captured prior to the installation of the corner reflectors resulted in only one natural persistent scatter on the bridge (by interpretation, this is likely to be from a metal lamp post). The processing of stacks of images collected after the corner installation identified new persistent scatterers, which can be attributed to the location of the corner reflectors. However, some of the corner reflector locations are missing persistent scatterer points. There are several potential reasons why this can be the case, such as a reflector becoming filled with snow or a bird nesting in the corner, which would reduce its reflectivity (although neither of these reasons appear to be the case in this study). If the corner was knocked out of alignment, or not aligned properly at installation, it would no longer face the line of sight of the satellite, and again, reduce in reflectivity.

Figure 6 Persistent scatterer (PS) points on Waterloo Bridge to compare to ATS prisms at the same locations (base map image from Open Street Map).

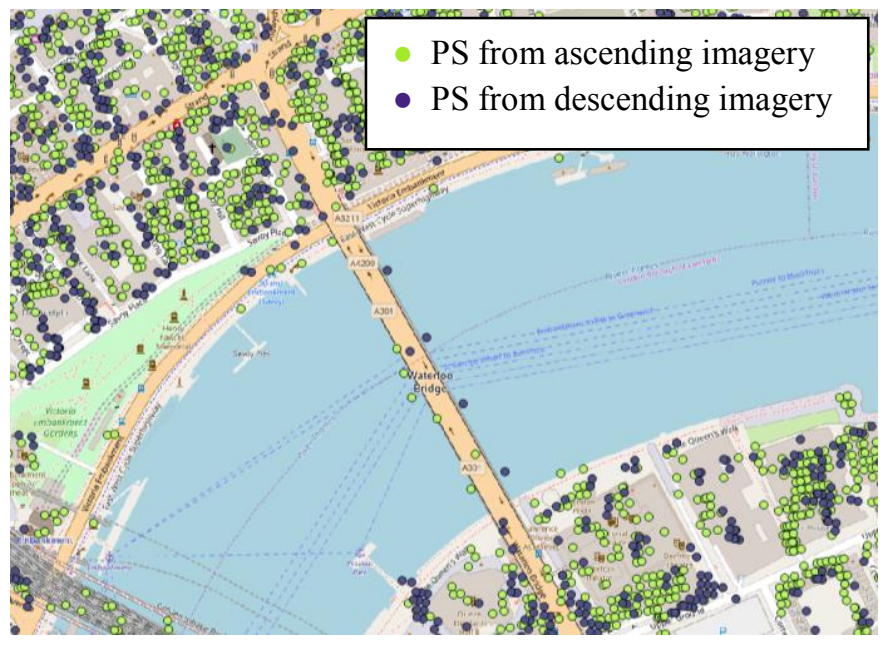


The results of interferometric processing are a change in displacement of each scatterer from one acquisition to the next, in the line of sight of the satellite. As such, the results provide a line of sight, change in movement reading at the 11-day intervals the images were acquired.

\subsection{Decomposition of InSAR for comparison with ATS}

Measurements in the satellite line of sight direction provide little structural interest, and the resolving of this vector in the vertical direction, longitudinal deck direction and transverse deck direction are considered. Using the SAR imaging geometry, the bridge alignment and one imaging direction (the west side corner reflectors only being visible to ascending aquisitions and the east side to descending) does not provide sufficient information to decompose the LOS measurement into movements in the desired longitudinal, transverse and vertical directions of the bridge.

Mathematically, the movement $\left(d_{L O S}\right)$ can be considered as Equation (1) below:

$\underline{A} \underline{y}^{T}=d_{L O S}$

Where

$\underline{A}=\left[\begin{array}{lll}\cos \theta & \sin \theta \cos \alpha & \sin \theta \sin \alpha\end{array}\right]$

$y=\left[\begin{array}{lll}d_{V} & d_{L} & d_{T}\end{array}\right]$

$\alpha$ is the heading angle relative to the bridge (marked on Figure 7 for ascending and descending directions), $\theta$ is the look angle (defined in Figure 1 and taken as $37.32^{\circ}$ for calculations), and $\mathrm{d}_{\mathrm{V}}, \mathrm{d}_{\mathrm{L}}$, and $\mathrm{d}_{\mathrm{T}}$ refer to the vertical, longitudinal and transverse components of the movement vector.

To form an initial comparison of datasets, a first approximation to assume all movement to be in the bridge longitudinal direction has been taken. With this initial assumption, the relationship between the line of sight (LOS) displacements and those in the bridge longitudinal direction was derived using the geometry in Figure 7.

Figure 7 Bridge orientation (plan view) in relation to satellite imaging geometry.

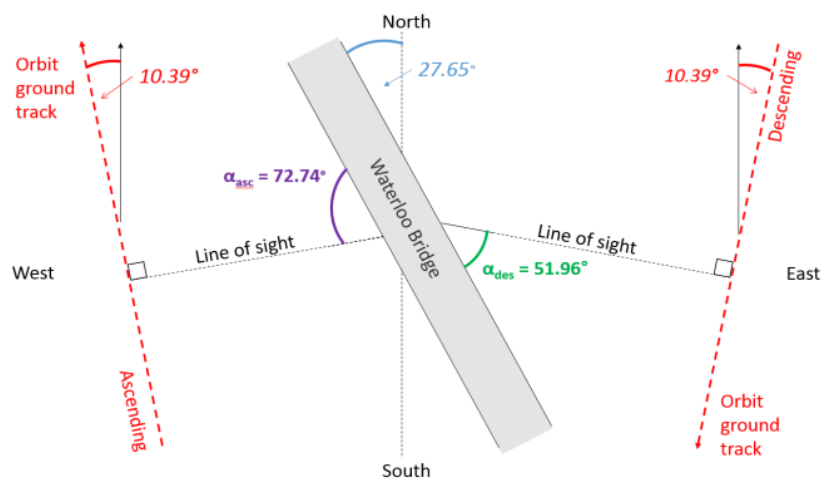

The flight path of SAR satellites is nearly north-south, making the system most sensitive to motion in an east-west direction, and almost unable to measure the displacement occurring in the north-south direction. This bridge has an orientation such that the SAR satellite is able to pick up a component of movement in the longitudinal direction of the bridge, but other bridges further up and down the river with north-south orientation would not be suitable for study.

The graph in Figure 8 plots the movement of the bridge when all line of sight of movement is assumed to be in the longitudinal direction. There is a notable anomalous point in April. As the images are not taken simultaneously, the SAR signal for each image can be affected differently by the atmosphere. The image in this instance is likely to have been affected by a significant atmospheric artefact and should be discarded from the readings.

The ATS readings, as described in section 5.1, have been used to measure longitudinal measurements relative to the south end of the bridge. In order to compare the two measurement systems, the longitudinal movements as relative to the southernmost reflectors were plotted. The results of one of these measurement sets (the difference between two pier measurements) is shown in Figure 9.

These plots of relative SAR measurements show good agreement with the corresponding relative movement between ATS prisms, but there is some variation on measurement at a single instant in time. The results of the experiment provide evidence that InSAR satellite readings can collect measurements in the order of magnitude required for bridge movements, and can follow the profile of seasonal movements. The decomposition assumptions used to facilitate this comparison both rely on assumptions that movement in other directions are limited, as well as increase the uncertainty of measurement and scaling errors through the vector transformation performed.

Additionally, it is worth noting that all relative movements showed good agreement between ATS and InSAR readings, with the exception of the values on either side of the end piers at the south end. The InSAR system suggests that this pier does not move and rebound with rise and fall of temperature as the others do. The values continue to move in one direction in the InSAR data. One possible suggestion is that the vertical component of the movement (ignored in the decomposition) does contribute more significantly to the line of sight reading, and in fact the bridge pier is settling. Analysis of the ATS relative vertical movements between piers suggests that this explanation can be discounted. This leaves an unexpected bridge behaviour effect that is not accounted for, and for which further monitoring or understanding using alternative methods could be employed to investigate the unusual behaviour flagged by InSAR readings to understand whether this is an actual behaviour of the bridge or a monitoring artefact. 
Figure 8 Graph of average bridge deck temperature and InSAR-measured longitudinal movement of points along the eastern side of the bridge plotted against time.

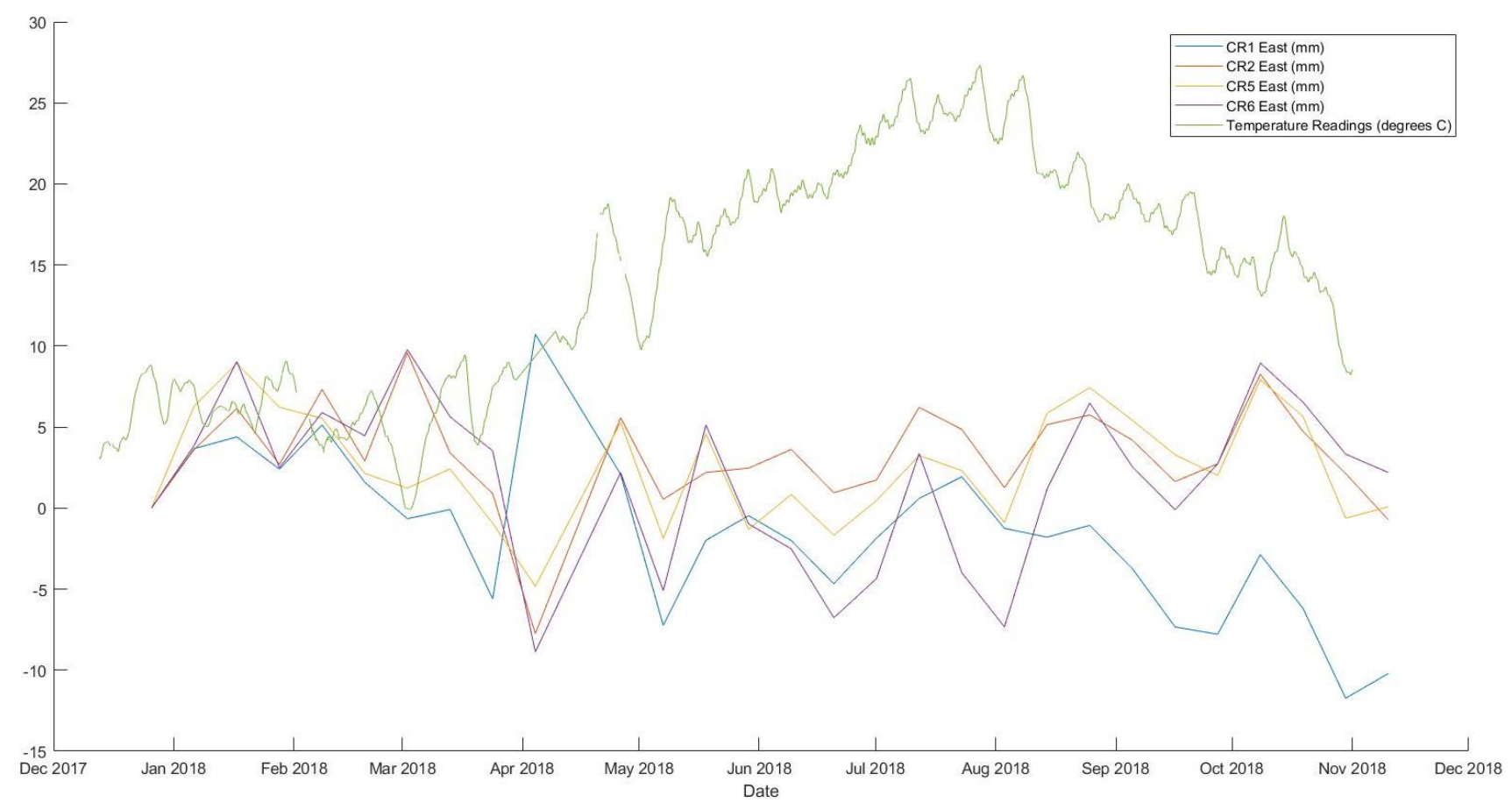

Figure 9 Plot of relative ATS and relative SAR movements between Piers 2 and 6 on the east side

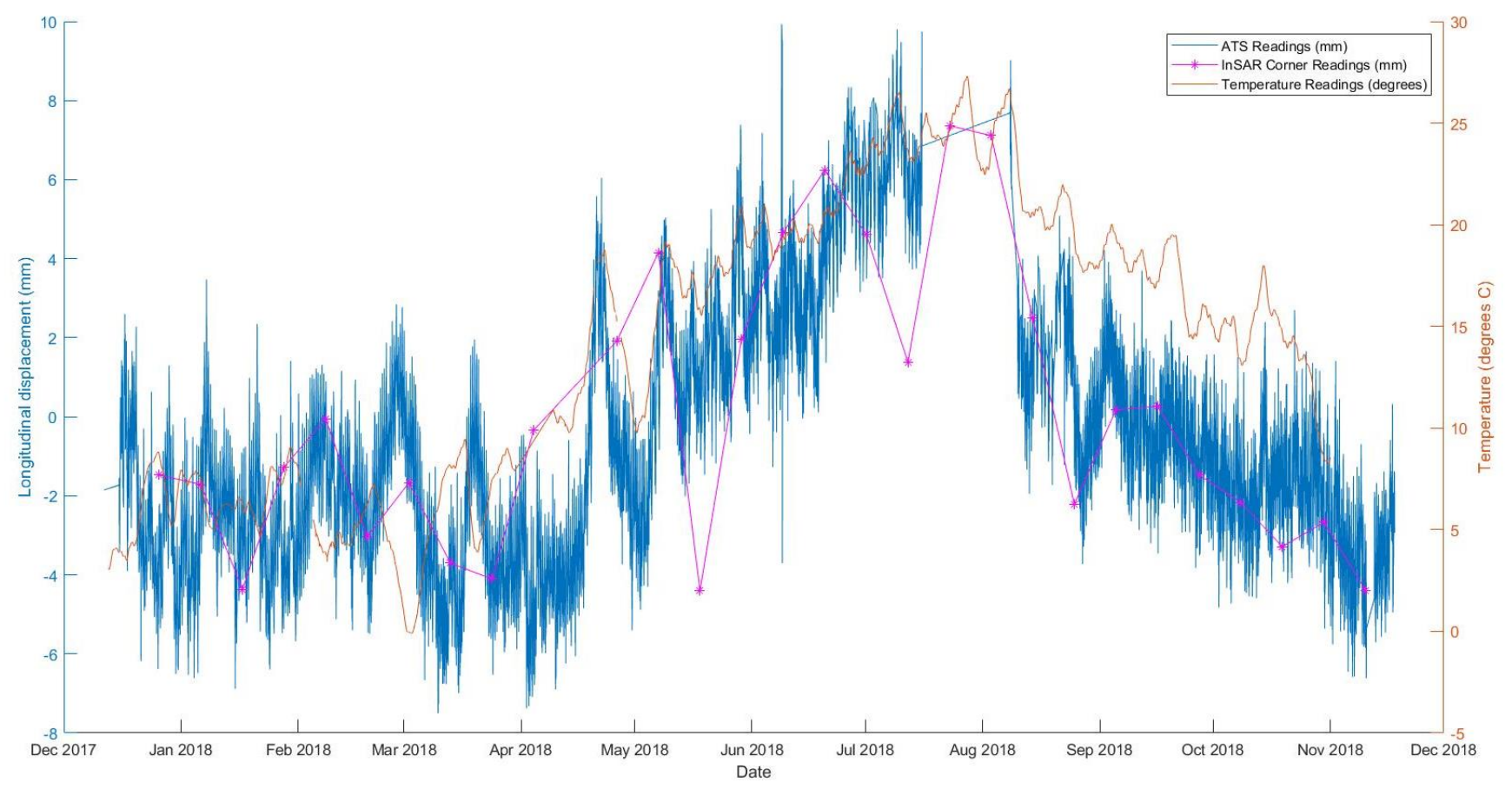




\subsection{Comparison with vertical displacement monitoring}

The vertical displacement derived from ATS data revealed very strong correlation with the height of tide. Although the results show readings within the right range of measurement, the frequency of satellite readings (at 11-day intervals) meant that there would be an insufficient number of data points over the time period to pick up the full tidal profile.

\section{Discussion and use of InSAR in structural health monitoring of bridges}

\subsection{Use of corner reflectors}

This project demonstrates the potential for bridge structures to be retrofitted with corner reflector devices to monitor points of interest over time using InSAR. However, there are two key considerations that have to be made in deciding whether to do so. Firstly, as previously outlined, the bridge must naturally have a low enough level of signal clutter such that the radar reflection from the corner reflector is clearly distinguishable from other points on the bridge.

Secondly, there must be a minimum spacing between the corners installed, such that the response from one corner does not interfere with another. This spacing is largely down to the resolution of the satellite imaging being used. In this case, reflectors spaced at distances greater than $24 \mathrm{~m}$ meant that $3 \mathrm{~m}$ resolution cells (which become larger than $3 \mathrm{~m}$ during the processing due to various filtering steps), would be clearly discernible from one another within the SAR radar imagery and interferometric processing.

\subsection{Limitations in bridge monitoring}

The key limitation is that the displacements measured are limited to the line of sight direction. To decompose this value into the desired directions, either some major assumptions have to be put in place, or three different satellite look directions would have to be employed (Wright et al., 2004). However, the reality of applying such methods into built environment applications is that, while the ascending and descending orbits pick up different sides of buildings, bridges, etc., this does not provide three different line of sight observations, at the same point in time, on the same physical point. In the specific application outlined in this paper, the different satellite passes (ascending and descending) each picked up different sides of the bridge, and as such the corner reflectors were designed and installed to face only the line of sight of the satellite that they were able to see. One other satellite radar system was available for this study, but this would have required another set of reflectors angled towards this additional satellite. The reflectors as installed did not pick up reflections from this third satellite direction.

Another limitation is the bridge surroundings. If the line of sight of the satellite is obscured (e.g. trees in the way) or blocked due to a shadow effect (e.g. by tall buildings in city centre locations), the bridge cannot be seen by the satellite for measurement, with or without natural or installed reflectors.
Additionally, the application of this technique is limited by bridge orientation (primarily direction of bridge movement). As the satellites travel in a near-polar orbit and are oblique looking (the line of sight images the ground to the east or west), any component of ground deformation in a north-south direction will not result in motion towards or away from the satellite. Therefore, movement in the north-south direction is not captured by InSAR, and as such longitudinal expansion of bridges with north-south orientation would not be picked up.

One further consideration is that the processing of InSAR data involves working out the $2 \pi$ ambiguity, and so large movements (larger than a half wavelength, in this particular case the full wavelength being $31 \mathrm{~mm}$ ) over short periods of time will not be captured. This measurement technique is only suited to slow moving, small scale deformations. The theoretical accuracy of InSAR is sub-millimetre (Ferretti et al., 2007b) but with a few millimetres of noise within the readings taken during this experiment and further uncertainty introduced through the process of decomposing the line of sight vector, InSAR measurement only becomes useful in structures that exhibit a scale of movement over 3 or $4 \mathrm{~mm}$ and under half of the radar wavelength $(15.5 \mathrm{~mm})$.

\subsection{Measurement assumptions and uncertainty}

The differential InSAR technique measures line of sight (LOS) changes of the relative displacement of a point in the SAR image with respect to a reference point. Within the processing chain, there is an opportunity to input points that are known to be motionless or whose displacement is known. Without input of these 'Ground Control Points' points are selected based on the imagery and assumed to be motionless or with known motion. The reference point is selected based on it having a high coherence value (an indication that the point is stable over time) and that it is not subject to local deformations. This means that the motion described by each of the points of interest on the bridge are affected by the actual motion of this point in a similar manner to the reference prisms for the ATS system influencing the reading of the ATS prisms on the bridge.

In this project the reference points were taken from large buildings within a couple of kilometres of the bridge site. These buildings appear to have little movement in the local area according to the SAR data supplied but could actually have some movement at the same millimetre scale as the deformation we are trying to measure on the bridge. As the 'absolute' movements taken by the satellite are actually relative to another point that is assumed to be fixed (which in practice will never truly be stationary) there will always be an unknown error in the 'absolute' measurements.

Based on this premise, it can be suggested that future work using a similar set up should include corner reflectors off the bridge, preferably fixed to the ground, that could be used as the datum for any movements. Further work considering practical implementation would be to consider if installing corner reflectors on a bridge and as datum points might be 
significantly less costly than installing and operating a traditional monitoring system over a period of years.

\subsection{InSAR historical archive}

Once an image is acquired, it can be saved for future, unforeseen, usage. A historic archive of imagery (if available over the area of interest and provided the asset of interest provides suitable coherent reflectors naturally, and without the need for corner reflectors) can be used to understand the behaviour of the asset and surrounding region years prior to the period of interest. Using archive imagery can also facilitate locating potentially suitable sites for "stable" areas for reference points (supporting traditional surveying methods).

\section{Conclusion}

The topic of InSAR time series measurement for the application of bridges was presented in this paper. Small scale corner reflectors were trialled and key points on the bridge were monitored over time using both InSAR and ATS measurements for comparison. Measurements were found to be comparable when comparing relative movement of points along the bridge from one another. The limited frequency of satellite readings and noise within readings means that it is not a technique that could replace traditional monitoring methods, but more seasonal trends can be tracked, and there is an opportunity to complement traditional inspection regimes with additional insights.

Future developments within the earth observation field could increase the sampling frequency of measurements. For example, putting more satellites in the same orbit enables shorter revisit times, which brings further opportunities for satellite monitoring. The current 11-day revisit period used in this study is only just about frequent enough to pick up trends within a season, and has a good chance of missing peak values or being affected by aliasing. If much shorter, say 3-hourly, readings were eventually possible with planned future satellite deployments, then the scope of observable behaviours would be much greater as loading patterns from diurnal temperature cycles, tidal motion, etc. would be picked up.

\section{Acknowledgements}

This work was made possible by EPSRC (UK) Award 1636878, with iCase sponsorship from the National Physical Laboratory and additional funding from Laing O'Rourke. TerraSAR-X satellite data was purchased by the Satellite Application Catapult through grant funding provided by Innovate UK.

The authors would like to express their thanks to Westminster City Council who have kindly provided permission and support for this research.

\section{References}

Bamler, R., Hartl, P., 1998. Synthetic aperture radar interferometry Synthetic aperture radar interferometry.
Inverse Probl. 14, 55. https://doi.org/10.1088/0266$5611 / 14 / 4 / 001$

Crosetto, M., Gili, J.A., Monserrat, O., Cuevas-González, M., Corominas, J., Serral, D., 2013. Interferometric SAR monitoring of the Vallcebre landslide (Spain) using corner reflectors. Nat. Hazards Earth Syst. Sci. 13, 923-933. https://doi.org/10.5194/nhess-13-923-2013

Crosetto, M., Monserrat, O., Cuevas-González, M., Devanthéry, N., Crippa, B., 2016. Persistent Scatterer Interferometry: A review. ISPRS J. Photogramm. Remote Sens. $115,78-89$.

https://doi.org/10.1016/j.isprsjprs.2015.10.011

Doerry, A.W., 2008. Reflectors for SAR Performance Testing, Sandia Report SAND2008-0396, Unlimited Release. Albuquerque.

Ferretti, A., Monti-Guarnieri, A., Prati, C., 2007a. InSAR Principles-Guidelines for SAR Interferometry Processing and Interpretation (TM-19). ESA 48.

Ferretti, A., Prati, C., Rocca, F., 2001. Permanent Scatters in SAR Interferometry. IEEE Trans. Geosci. Remote Sens. 39, 8-20. https://doi.org/10.1109/36.898661

Ferretti, A., Prati, C., Rocca, F., 2000. Nonlinear subsidence rate estimation using permanent scatterers in differential SAR interferometry. IEEE Trans. Geosci. Remote Sens. 38, 22022212. https://doi.org/10.1109/36.868878

Ferretti, A., Savio, G., Barzaghi, R., Borghi, A., Musazzi, S. Novali, F., Prati, C., Rocca, F., 2007b. Submillimeter accuracy of InSAR time series: Experimental validation. IEEE Trans. Geosci. Remote Sens. 45, 1142-1153. https://doi.org/10.1109/TGRS.2007.894440

Fornaro, G., Reale, D., Verde, S., 2012. Monitoring thermal dilations with millimetre sensitivity via multi-dimensional SAR imaging. Proc. 2012 Tyrrhenian Work. Adv. Radar Remote Sens. From Earth Obs. to Homel. Secur. TyWRRS 2012 131-135.

https://doi.org/10.1109/TyWRRS.2012.6381117

Garthwaite, M.C., Nancarrow, S., Hislop, A., Thankappan, M., Dawson, Lawrie, J.H., 2015. The Design of Radar Corner Reflectors for the Australian Geophysical Observing System. https://doi.org/ISSN 2201-702X

Garthwaite, M.C., Thankappan, M., Williams, M.L., Nancarrow, S., Hislop, A., Dawson, J., 2013. Corner reflectors for the Australian Geophysical Observing System and support for calibration of satellite-borne synthetic aperture radars. Int. Geosci. Remote Sens. Symp. 266-269. https://doi.org/10.1109/IGARSS.2013.6721143

Gentile, C., Bernardini, G., 2008. Output-only modal identification of a reinforced concrete bridge from radarbased measurements. NDT E Int. 41, 544-553. 
https://doi.org/10.1016/j.ndteint.2008.04.005

Lazecky, M., Hlavacova, I., Bakon, M., Sousa, J.J., Perissin, D., Patricio, G., 2017. Bridge Displacements Monitoring Using Space-Borne X-Band SAR Interferometry. IEEE J. Sel. Top. Appl. Earth Obs. Remote Sens. 10, 205-210. https://doi.org/10.1109/JSTARS.2016.2587778

Sarabandi, K., Tsen-Chieh Chiu, T.-C., 1996. Optimum corner reflectors for calibration of imaging radars. IEEE Trans. Antennas Propag. 44, 1348-1361.

https://doi.org/10.1109/8.537329

Selvakumaran, S., Plank, S., Geiß, C., Rossi, C., Middleton, C., 2018. Remote monitoring to predict bridge scour failure using Interferometric Synthetic Aperture Radar (InSAR) stacking techniques. Int. J. Appl. Earth Obs. Geoinf. 73, 463470. https://doi.org/10.1016/j.jag.2018.07.004

Simons, M., Rosen, P. a, 2007. Interferometric Synthetic Aperture Radar Geodesy. Treatise Geophys. 3, 391-446. https://doi.org/10.1016/B978-0-12-386874-9.00010-5

Sousa, J.J., Bastos, L., 2013. Multi-temporal SAR interferometry reveals acceleration of bridge sinking before collapse. Nat. Hazards Earth Syst. Sci. 13, 659-667. https://doi.org/10.5194/nhess-13-659-2013

Webb, G.T., Vardanega, P.J., Middleton, C.R., 2014. Categories of SHM Deployments : Technologies and Capabilities. J. Bridg. Eng. https://doi.org/10.1061/(ASCE)BE.1943-5592.0000735.

Wright, T.J., Parsons, B.E., Lu, Z., 2004. Toward mapping surface deformation in three dimensions using InSAR.

Geophys. Res. Lett. 31, 1-5.

https://doi.org/10.1029/2003GL018827 